\title{
ON A QUESTION OF FEIT
}

\author{
PAMELA A. FERGUSON ${ }^{1}$ AND ALEXANDRE TURULL
}

\begin{abstract}
The following theorem is proved: Assume $\chi$ is an irreducible complex character of the finite group $G$ and $G$ is $\pi$-solvable where $\pi$ is the set of prime divisors of $\chi(1)$. Then $G$ contains an element of order $f(\chi)$.
\end{abstract}

Introduction. All groups in this paper are finite. The question referred to in the title is the following: Let $\chi$ be an irreducible complex character of a finite group $G$ and let $f(\chi)$ be the smallest positive integer such that $\{\chi(g) \mid g \in G\} \subseteq Q(\alpha)$ where $\alpha$ is a primitive $f(\chi)$ th root of unity. Does $G$ contain an element of order $f(\chi)$ ?

By using factorizations of quasi-primitive irreducible characters into products of characters we obtain the following result:

THEOREM. Assume $\chi$ is an irreducible complex character of a finite group $G$ and $G$ is $\pi$-solvable where $\pi$ is the set of primes dividing $\chi(1)$. Then $G$ contains an element of order $f(\chi)$.

This theorem yields the following Corollary which was proved independently by Amit and Chillag [1].

COROLLARY. If $\chi$ is an irreducible character of a solvable group $G$, then $G$ contains an element of order $f(\chi)$.

We wish to thank Professor I. M. Isaacs and the referee for carefully reading the original manuscript and making helpful suggestions. In particular, the notation $f(\chi)$ was suggested by Professor Isaacs in honor of W. Feit.

1. We introduce some definitions and notation. Let $\sigma$ be a nonempty set of primes and $h$ be an element in a group $G, h$ is a $\sigma$-element if $\langle h\rangle$ is a $\sigma$-group. Any $g \in G$ may be written uniquely as $g=g_{\sigma} g_{\sigma^{\prime}}$, where $g_{\sigma}$ is a $\sigma$-element, $g_{\sigma^{\prime}}$ is a $\sigma^{\prime}$-element and both $g_{\sigma}$ and $g_{\sigma^{\prime}}$ are powers of $g$. The elements $g_{\sigma}$ and $g_{\sigma^{\prime}}$ are called the $\sigma$-part and $\sigma^{\prime}$-part of $g$.

If $\Omega$ is a Galois extension of the field $\Omega_{1}, G\left(\Omega / \Omega_{1}\right)$ denotes the Galois group of $\Omega$ over $\Omega_{1}$.

If $\chi \in \operatorname{Irr}(G)$ and $\sigma$ is a set of primes, then $\chi$ is $\sigma$-special provided that $\chi(1)$ is a $\sigma$-number and that for all subnormal subgroups $S$ of $G$ and all irreducible constituents $\theta$ of $\chi_{S}$, the determinantal order $O(\theta)$ is a $\sigma$-number.

PROOF OF THEOREM. The result is clear for linear characters so by induction on $\chi(1)$ we assume that the theorem is true for irreducible characters $\Phi$ and groups $H$ satisfying the hypothesis if $\Phi(1)<\chi(1)$. If $\chi=\Phi^{G}$ where $\Phi \in \operatorname{Irr}(H)$ and

Received by the editors January 30, 1985 and, in revised form, May 1, 1985.

1980 Mathematics Subject Classification. Primary $20 \mathrm{C} 15$.

Key words and phrases. Prime character, $\pi$-special character, $f(\chi)$.

${ }^{1}$ Partially supported by a National Science Foundation Grant. $0002-9939 / 86 \$ 1.00+\$ .25$ per page 
$|H|<|G|$, then $f(\chi) \mid f(\Phi)$. Since $\Phi(1)$ is a proper divisor of $\chi(1), H$ contains an element of order $f(\Phi)$ and the theorem follows. Thus, we may assume that $\chi$ is primitive.

Let $f(\chi)=\prod_{i=1}^{n} p_{i}^{a_{i}}$ where $a_{i} \geq 1$ and the $p_{i}$ are distinct primes. Let $p_{i}^{r_{i}}$ denote the order of a Sylow $p_{i}$-subgroup of $G$ for $i=1, \ldots, n$. Then $\Omega_{i}$ denotes the field of $\left(|G| / p_{i}^{r_{i}-a_{i}+1}\right)$ th roots of unity over $Q$. Let $\Omega$ be a field of $|G|$ th roots of unity over $Q$. By the definition of $f(\chi)$, we may choose a $\theta_{i} \in G\left(\Omega / \Omega_{i}\right)$ such that $\theta_{i}$ does not leave $\chi$ invariant for $i=1, \ldots, n$. We first show that no product of an odd number of distinct elements in $\left\{\theta_{1}, \ldots, \theta_{n}\right\}$ leaves $\chi$ invariant. Assume otherwise; then since $G(\Omega / Q)$ is abelian, we may choose notation so that $\beta=\prod_{i=1}^{r} \theta_{i}$ leaves $\chi$ invariant, for some $r \geq 1$.

Suppose $p_{i} \mid \chi(1)$ for some $i=1, \ldots, r$. Since $G(\Omega / Q)$ is abelian, we may assume $p_{1} \mid \chi(1)$. By [4, Corollary 2.7], $\chi=\chi_{1} \Phi$ where $\chi_{1}$ and $\Phi$ are primitive irreducible $p_{1}$-special and $p_{1}^{\prime}$-special characters. $G$ is $p_{1}$-solvable, hence $p_{1}^{\prime}$-separable, and so $f(\Phi)$ is a $p_{1}^{\prime}$-number by $[3$, Proposition $6.3(\mathrm{a})]$.

If $\theta \in G(\Omega / Q)$ and $\sigma$ is any set of primes, then it is direct to see that whenever $\gamma$ is an irreducible $\sigma$-special character so is $\theta \circ \gamma$. Hence $\beta \circ \chi_{1}$ and $\beta \circ \Phi$ are $p_{1}$-special and $p_{1}^{\prime}$-special. Now $\chi_{1} \Phi=\chi=\beta \circ \chi=\left(\beta \circ \chi_{1}\right)(\beta \circ \Phi)$ and [4, Theorem 2.2] yield $\chi_{1}=\beta \circ \chi_{1}$. By [3, Proposition 6.3(a)], $f\left(\chi_{1}\right)$ is a $p_{1}$-number so $\theta_{i} \circ \chi_{1}=\chi_{1}$ for $i=2, \ldots, r$. Since $G(\Omega / Q)$ is abelian, it follows that $\chi_{1}=\beta \circ \chi_{1}=\theta_{1} \circ \chi_{1}$. Now $f(\Phi)$ a $p_{1}^{\prime}$-number implies that $\theta_{1} \circ \Phi=\Phi$. However, $\theta_{1} \circ \chi=\left(\theta_{1} \circ \chi_{1}\right)\left(\theta_{1} \circ \Phi\right)=\chi_{1} \Phi=\chi$ contradicts the choice of $\theta_{1}$. Therefore, $\left(\prod_{i=1}^{r} p_{i}, \chi(1)\right)=1$.

Now set $p=p_{1}$. By $[4$, Corollary 2.7], $\chi=\Phi \lambda$, where $\Phi$ and $\lambda$ are irreducible, primitive $\pi$-special and $\pi^{\prime}$-special characters. Since $\chi(1)$ is a $\pi$-number, $\lambda$ is linear. As in the previous paragraph, $\beta \circ \Phi$ and $\beta \circ \lambda$ are $\pi$-special and $\pi^{\prime}$-special. Thus, $\Phi \lambda=\chi=\beta \circ \chi=(\beta \circ \Phi)(\beta \circ \lambda)$ and $[4$, Theorem 2.2] imply that $\lambda=\beta \circ \lambda$. Since $\beta$ fixes $\lambda, \beta$ fixes all powers of $\lambda$. In particular, $\beta$ fixes $\lambda_{p}$ and $\lambda_{p^{\prime}}$, where $\lambda_{p}$ is the $p$-part of $\lambda$ and $\lambda_{p^{\prime}}$ is the $p^{\prime}$-part. It is clear that $f\left(\lambda_{p}\right)$ is a $p$-number and $f\left(\lambda_{p^{\prime}}\right)$ is a $p^{\prime}$-number. Hence, $\theta_{i} \circ \lambda_{p}=\lambda_{p}$ for $i=2, \ldots, r$ and $\theta_{1} \circ \lambda_{p^{\prime}}=\lambda_{p^{\prime}}$. Since $G(\Omega / Q)$ is abelian, $\lambda_{p}=\beta \circ \lambda_{p}=\theta_{1} \circ \lambda_{p}$. By [3, Proposition 6.3(a)], $f(\Phi)$ is a $\pi$-number. Thus, $\theta_{1} \circ \Phi=\Phi$ and $\theta_{1} \circ \chi=\left(\theta_{1} \circ \Phi\right)\left(\theta_{1} \circ \lambda_{p}\right)\left(\theta_{1} \circ \lambda_{p^{\prime}}\right)=\Phi \lambda_{p} \lambda_{p^{\prime}}=\chi$. Again this is a contradiction. Therefore, no product of an odd number of distinct $\theta_{i}$ leaves $\chi$ invariant. Hence, by [2, Theorem 2], there is an element $g \in G$ such that $\chi(g) \notin \Omega_{i}$ for any $i=1, \ldots, n$. Thus, $f(\chi)||\langle g\rangle \mid$.

\section{REFERENCES}

1. G. Amit and D. Chillag, On a question of Feit, Pacific J. Math. (to appear).

2. R. Brauer, A note on theorems of Burnside and Blichtfeldt, Proc. Amer. Math. Soc. 15 (1964), 31-34.

3. D. Gajendragadkar, A characteristic class of characters of finite $\pi$-separable groups, $\mathrm{J}$. Algebra 59 (1979), 237-259.

4. I. Isaacs, Primitive characters, normal subgroups, and M-groups, Math. Z. 177 (1981), 267-284.

Department of Mathematics, University of Miami, Coral Gables, Florida 